\title{
Summary of Satellite Collaborative Planning Algorithm
}

\author{
Jie Zhang, Gang Wang, Fangzheng Zhao, and Guiling Tian
}

\begin{abstract}
In the past, the way to manage and control satellite operations was mainly accomplished by planning the mission plan in advance through the ground. However, the current satellite missions are becoming more and more complex, and it is necessary to face sudden emergency situations and changes in the operating environment. The management model presents many challenges. This paper takes the single-satellite collaborative planning as the satelliteting point, and summarizes and forecasts the planning research of the satellite collaborative algorithm, which focuses on the research status of satellite autonomous and collaborative task scheduling planning, and summarizes the research progress of single-star and multi-star mission scheduling problems according to "ground offline - on-line online - satellite integration". In a word, satellite autonomous and collaborative task scheduling has broad research prospects.
\end{abstract}

Index Terms - Independent collaboration, satellite, integration of satellite and earth.

\section{RESEARCH ON A SingLe SATELLITE INDEPENDENT Collaborative PlanNing AlgORITHM}

At present, the research and application of domestic satellite mission scheduling planning mainly focuses on the ground management and control mode. The research on the on-board autonomous mission scheduling is still in its infancy. The main task scheduling research is based on single star and further extended to the multi-star collaborative framework. This paper focuses on the research of satellite autonomous and collaborative scheduling planning, and combs and analyzes the research status of single-star autonomous task scheduling planning according to the structure of "single star ground offline - single star online satellite integration", according to "multi-star ground". The offline-multiple stars are online - multi-star integration structure analyzes and analyzes the current status of multi-satellite collaborative task scheduling planning, and finally summarizes satellite autonomous and collaborative scheduling planning research.

With the continuous increase of satellite payload capacity, the satellite response time requirements are continuously shortened. In order to alleviate the limited status of satellite resources and improve the satellite's ability to respond quickly to emergencies, the most realistic approach is to give satellites independent decision-making and independent communication coordination. The ability to self-schedule planning enables satellites to perform autonomous mission

Manuscript received September 18, 2019; revised January 31, 2020.

Jie Zhang, Fangzheng Zhao and Guiling Tian are with the Graduate School, Air Force Engineering University, Xi'an 710054 China (e-mail: afeu_zhangjie@163.com, zhaofz1020@163.com, david liszt tian@163.com).

Gang Wang is with the Department of Air-Defense and Anti-Missile, Air Force Engineering University, Xi'an 710054 China (e-mail: iamwg@163.com). planning based on dynamic environments. The on-board autonomous planning capability enables satellites to no longer rely entirely on external control information, based on dynamically arriving observational requirements, real-time climatic conditions, and satellite operational status. Perform on-board online planning and generate action instructions in orbit. The ability of inter-satellite collaborative planning allows collaborative requirements to reach designated satellites directly through the inter-satellite link without the ground. Autonomous and collaborative planning techniques can be assumed by the ground system. Planning, control and other functions are transferred to the satellite. Not only can the scale of the ground station be reduced, the operating cost can be saved, the information interaction between the stars and the ground can be reduced, and more importantly, the dynamic observation demand can be responded to in time, and a wide range can be realized through multi-star cooperation, continuous observation and monitoring throughout the day, all day. The target discovery is confirmed. At the same time, the satellite's independent planning considers the real-time status of the satellite, so it can realize the optimal allocation of the resources on the star, and then can promote the long-term stable operation of the satellite. Thus, satellite autonomy and coordination. The research heat of task scheduling planning is increasing day by day.

\section{A. Single-Satellite Offline Task Scheduling}

The single-satellite ground offline mission planning can be generally divided into four phases. The task pre-processing phase mainly analyzes the user requirements and studies the task decomposition and clustering methods. The model construction phase mainly determines the objective function according to the formal requirements, under consideration of constraints. Modeling the mission planning objectives, designing the algorithm phase to study the algorithm for solving the satellite programming problem, and evaluating the performance of the algorithm and the advantages and disadvantages of the scheme in the evaluation phase. In order to be able to complete the complex processes in these phases, the computing unit has higher computing power requirements. Conventional task scheduling planning generally chooses the method of ground offline. In the task preprocessing stage, in order to improve the utilization of scarce satellite resources, the related research first considers the decomposition and clustering of satellite missions. $\mathrm{Wu}$ [1] proposed a two-stage scheduling. The method first obtains the clustering task by using the improved minimum group partitioning algorithm, and then implements a hybrid ant colony algorithm based on the overall task and the obtained cluster task to achieve local optimal or approximate optimal scheduling, for this static clustering. The shortcomings of the strategy, follow-up research proposed a dynamic task clustering strategy [2], also research User needs, proposed plan [3] based on user demand for more multi-objective indicators of local search methods, 
so as to enhance the overall effectiveness of resources. Model building phase in phase

The study of the task scheduling problem from multiple aspects such as: graph theory problem model [4]-[6], knapsack problem model [7], [8], integer programming model [9], [10] and other models [11], [12] Modeling. The design algorithm phase generally solves the satellite mission planning scheme under the conditions of storage capacity and time window constraints [13].

\section{B. Single Satellite Online Task Scheduling Planning}

Compared to ground-based offline, the on-board online mission scheduling plan requires satellites to have certain autonomy: 1) Improve the ability to respond to emergencies, such as forest fires, volcanic eruptions, ship detections, and images that are completely obscured by clouds. Invalidation, satellite autonomous task scheduling can directly trigger secondary imaging for the same target point. For images with cloud coverage and fire detection tasks, images with no fire points can be deleted immediately on the satellite to free up storage space; 2) Quick response to task conflicts, can be used for other low-priority tasks and unscheduled tasks; 3) Improve satellite operational efficiency, task scheduling can take into account the precise state of the onboard resources, such as current instrumental heat, solar panels The power and the remaining space of the on-board memory further enhance the effectiveness of the mission; 4) Reduce the reliance on ground monitoring and control, so that the satellite does not have to maintain frequent communication with the ground, and enhance the robustness of the satellite independent operation. Constrained by on-board computing power, the constraints of on-line online task scheduling planning are more complicated. Related research is mainly carried out from planning methods and strategies [14], Chien [15] based on domain knowledge-based iterative repair planning strategy proposed onboard autonomous architecture And expanded, Khatib used a forward heuristic greedy search algorithm to explore the on-board autonomous planning, Damiani introduced the dynamic programming algorithm into the on-board autonomous scheduling, and Beaumet proposed an iterative random greedy search algorithm. To calculate and process the satellite's state, task priority, imaging time and energy demand, and realize the independent planning of cloud detection capability. Sa [13] proposed a real-time fault-tolerant algorithm based on Hopfield neural network for real-time fault-tolerant algorithm. For the autonomous response of emergency tasks, Baek proposed an improved genetic algorithm and designed a GUI to improve the overall benefit of satellite autonomous task scheduling. Sun proposed a new scheduling algorithm based on genetic algorithm to solve the problem of satellite autonomous mission scheduling. Kono uses a Store a knowledge base of historical flight operations plans to guide satellite autonomous mission rules Tian Zhixin uses real-time telemetry to obtain the dynamic constraint parameters of the task running process, and generates the task instruction sequence online based on the directed graph model. In addition, the on-line online task scheduling planning requires fast, efficient and algorithmic convergence. The sequential heuristic algorithm optimizes the algorithm by mixing multiple rules. Kim realizes the rapid processing and target allocation of SAR satellite formation after receiving the user observation request, and designs a fast solution method based on genetic algorithm. Herz introduced The Orb View mission system, which integrates a high-fidelity aircraft simulator, uses a variety of optimization algorithms and predefined schedules to quickly derive optimal imaging schemes under coordinated constraints. The following table shows the current single-satellite online mission scheduling system. Perform combing analysis.

\section{SUMMARY AND OUTLOOK}

In summary, satellite autonomous and collaborative task scheduling has broad research prospects, and its possible future development directions are:

1) Collaborative task scheduling for large-scale satellite clusters [16]. The distributed Earth observation satellite system currently used is small in scale. With the expansion of satellite scale and the increase of payload types, a single control structure will inevitably affect satellite clusters. Collaborative efficiency, fully distributed constellation structure will be the development direction of largescale satellite cluster cooperative mission planning. In future research, how to dynamically respond to a specific task demand in a large-scale satellite cluster, synergistic multi-load Completing the multi-method investigation of the target and maintaining the stable operation of the constellation has become a key issue in the study of cooperative task scheduling for large-scale satellite clusters.

2) Close to the satellite scheduling plan that fully considers constraints. Most studies only carry out hypothesis experiments for a particular scenario, without considering many constraints in practical engineering applications, such as: regional target/moving target observation window changes; on-board energy Uncertainty with solid storage; communication time window and communication loop constraints on communication. With the development of satellite technology in the future, it is required to implement fine-grained scheduling for satellites. The determination of specific time points needs to consider the scheduling of corresponding resources. And modeling and solving these scheduling sub-problems together, this requirement of completely considering constraints has brought a new direction for satellite scheduling planning research. In addition, the onboard computing power is limited, how to improve the algorithm under the consideration of complex constraints Speed and efficiency are also the next research directions.

Introducing on-board autonomous mission planning for machine learning. When performing a mission, the autonomous satellite generates a large number of data such as Earth observation actions, data backhaul actions, and daily directed actions. The machine learning module can be introduced to parse the data., allowing satellites to improve the performance of autonomous planning modules by automatically acquiring knowledge. Combining machine learning with autonomous satellite mission planning involves key information extraction, state monitoring and comprehensive assessment of the environment and satellite 
itself, based on historical data and decision models. Adjusting the scheduling strategy and other research issues. The future on-board computer capabilities will be gradually strengthened, and the autonomous satellite application scenarios will be widened. There will be higher research requirements for the decision-making ability and mission planning capability of intelligent autonomous satellites.

\section{Multi-STAR INTEGRATED TASK SCHEDULING}

The multi-satellite collaborative task scheduling plan for satelliteintegrated integration mostly adopts the centralized-distributed architecture. First, the ground management is the central entity of the whole system. Through the latest information on the on-board status and task attributes, task assignment is performed in each local entity. And in the visible time window, the initial planning plan is released to each satellite. Each satellite acts as a local entity, taking into account its own status, task requests/recommendations of the central entity, and emergencies, and coordinating with the constellation. Planning. The satelliteintegrated architecture not only utilizes the powerful computing power of the ground, but also takes into account the advantages of autonomous on the star. For example, the autonomous decision on the star can obtain the state information of the satellite itself in real time, but the ground control station cannot; New ground conditions and timely response to detected conditions; on-board autonomous cloud judgments enable satellites to avoid useless visible light imaging activities; on-board image processing analysis enables satellites to first remove useless observed image data and then save To the storage space on the star, to avoid useless data downlink. DIPS system (Distributed intelligent Planningand scheduling) is a distributed intelligent planning and scheduling system. DIPS-based satellites only receive high-order targets from ground stations, perform autonomous scheduling and planning on the satellite, and cooperate with other satellites to complete the target mission. It will each satellite. Modeled as an agent with autonomous functions, and adopted a domain allocation algorithm, which usually creates a feasible scheduling scheme after the first coordination work, thereby reducing the negotiation between agents in the scheduling process, while maintaining constraints and On the basis of constraints, according to the constraint propagation paradigm, the optimization agent is used to optimize the use of time and resources, realize prioritybased scheduling, and use a hierarchical confirmation/authorization mechanism between agents to achieve global target coordination.

Aiming at the on-board autonomous planning and scheduling module, a set of star-ground integrated multi-star collaborative mission planning scheme is proposed, in which the centralized distributed decision-making architecture is composed of two parts: one is centered on the ground control station. The tracking task allocation mechanism will be triggered frequently. Second, each satellite has an on-board reactive decision/planning mechanism, which is always in working state and can be interrupted at any time. Tripp proposed a task management system based on Agent indirect communication coordination. Centralized-distributed architecture, in which the ground station publishes a series of mission requests to all satellites at once, and then each satellite is marked by other satellites as a set of tasks to be executed and in the last round according to their own workload. Repeat the task set and so on, select the tasks that can be performed.

In the context of the Global Environment and Security Monitoring Program (GMES), Cesta conducts research on distributed agents for autonomy (DAFA) technology and demonstrates the advantages of multi-agent technology in satellite applications, aiming at the Agent-based design framework. Define, design and develop the Agent system; Grey for the GMES project, build a Holistic Agent Space Architecture (HASA) for the multi-agent system, which includes four types of Agents: Product, Executor, Planner and Resource. The spatial distribution and the task type are divided into two dimensions, and the agents are divided as follows: Ground segments for multi-star collaborative tasks, including user priority definition modules, task assignment modules and ground stations; ground segments for single-star missions, including Flight dynamics module, data processing module, task planner and task priority definition module; space segment for single-star tasks, including on-board planning module, on-board communication module, on-board actuator, on-board monitoring, on-board planning Scheduler and on-board detection payload.

\section{Multi-STAR ONLINE COLlabORATIVE TASK SCHEDULING}

Most of the research simplifies the multi-satellite collaborative task scheduling problem to the optimization problem, and solves it with traditional optimization methods such as greedy, backtracking and simple heuristics. These studies generally only consider small-scale problems and cannot meet the needs. The need for multi-satellite collaborative task scheduling for dynamic task environments Similar to single-star, multi-star mission scheduling also requires the placement of on-board processing to cope with the more complex dynamic environment of multiple stars, the difference being that multiple stars are still It is required to achieve inter-satellite collaboration. For conservative reasons, online collaborative task scheduling for multi-stars generally adopts a centralized architecture. For example, NASA uses a spacecraft in the satellite formation as the parent star in the ASE environment to schedule the companion through inter-satellite communication. The operation of the spacecraft $\mathrm{Tl}$ proposes a spacecraft constellation interaction scenario, constructing a fully centralized architecture with high-orbit satellites as the main star and low-orbit satellites as member stars. The constellation consists of 16 low-orbit sun-synchronous satellites and 4 highs. Orbiting satellites (the fourth of which acts as a backup star), in which the high-orbiting satellite receives resource status information sent by the low-orbit member stars. The global behavior is controlled. The onboard computer has powerful processing capability, is responsible for the management of the entire constellation, and maintains communication interaction with the ground control station at all times. The low-orbit satellite is relatively simple, and receives commands and changes from the high-orbit satellite to the mission target. And transmit its own resource status data to the high-orbiting 
satellite. Zheng uses the deep-space low-frequency electromagnetic wave detection project as the background, allowing the main star to load the mission planning work of all satellites in the whole system, using adaptive genetic algorithm to decompose the mission target and complete the task of the constellation. Planning and scheduling, simulation experiments show that this fully centralized planning system can obtain a better scheduling solution in short-term planning Qin targets heterogeneous low-orbit small satellite networks, adopts a fully centralized architecture, and proposes resources based on deadlines. Deadline based resource balancing (DBRB). The DBRB algorithm is configured on the main star of the satellite cluster. The main star is required to maintain the latest state of each member star. The remaining power supply of each member star and the loaded load type are selected. Hou Xuanxing, task execution. But centralized architecture for the main star Computational power and inter-satellite communication link requirements are high, and once the main star fails, the overall system performance will be greatly compromised. In order to cope with the more complex communication environment and reduce the dependence on the central satellite, the decentralized distributed scheduling method is subject to Fortunately, Bonnet proposed a decentralized architecture and corresponding hybrid optimization method for the ground observation star task scheduling of the Fuego project: For single-star planning, adopt a cautious and heavy method, generate plans on the star, and set the task request and execution intent. Sharing to other satellites; for multi-satellite coordination, using reactive rules, different conflict resolution strategies and steps for soft and hard conflicts caused by redundant allocation, follow-up study

Considering the distributed task assignment problem in distributed satellite systems considering the asynchronous arrival and intermittent communication of mission requests, an online progressive dynamic organization mechanism is proposed, which is represented by the following three steps: Independent planning of each satellite; Communication, building common knowledge; complex tasks require multiple satellites to achieve different subtasks to complete, then each satellite constructs and modifies the alliance formation. The communication constraints in this application scenario are: Ground control station only communicates with high orbit satellites High-orbiting satellites act as relays between themselves, ground stations and low-orbiting satellites; low-orbit satellites can only communicate with highorbiting satellites. Horst studies mission-distribution problems for distributed satellite systems, using full distribution Market auction mechanism to effectively allocate tasks in unstable networks without global state knowledge. Each member star calculates the bid value according to the remaining level of resources, and uses its relative fitness as the bid value, and the task. Assigning stars for communication. Bonnet uses a multi-target adaptive multiagent system for solution optimization. For the scheduling problem, the system adopts the decentralized distributed scheduling method to solve the dynamic scheduling problem of the constellation. Each satellite obtains the consensus opinion with other satellites through local scheduling and multiple cooperative communication. Zhang Zhengqiang will discuss the distributed satellite system mission planning and the control problem is divided into two levels: constellation task planning and single-star autonomous control, and then a high-reliability centralized-distributed architecture is established. The hierarchical hybrid structure of the imaging satellite agent is constructed, including the global control layer, communication and the five layers of the collaboration layer, the local planning layer, the monitoring layer and the execution layer. On the basis of the above framework, a constellation mission planning method is proposed, which is based on the constrained honest contract network protocol. Strategy and bid evaluation strategy. Gao Li has carried out research on task cooperation mechanism from two aspects of negotiation and task optimization allocation, and expanded the traditional contract network protocol to introduce intelligent mental state parameters such as trust degree and cooperation frequency. Pang Zhonghua from Low-orbit satellite online mission planning has high timeliness requirements and high conflict adjustment requirements

Starting with the three characteristics of resource types, the traditional centralized-distributed architecture is extended. A class of middle management agents is added to the main star and member stars to form a multi-agent architecture with three types of agents.

\section{CONCLUSION}

This paper reviews the research status of satellite autonomous and collaborative task scheduling planning, and summarizes the research progress of single-star and multi-star mission scheduling problems according to "ground offline - on-line online - satellite integration". There are already many valuable research results in the field, and related research has been widely concerned by scholars all over the world. But it is not difficult to find that many of the current research results have their specific application background, so it is inevitable that there are certain defects: 1) Single-star online scheduling research is mostly for rescheduling of a single emergency task that is randomly arrived or generated by itself, and in the face of an increasingly dynamic emergency mission environment, where there are multiple sources of emergency tasks, including ground emergency betting, Hexing communication and its own emergency generation, etc., there is a need for a single-star online scheduling mechanism and method that takes into account both emergency response performance and limited on-board computing conditions. 2) Most studies will consider autonomous and collaborative task scheduling problems. A number of simplifications and assumptions are made to the research object, such as: Full communication or stability between stars in a distributed architecture Link; on-board computing power reaches a high level, computing time meets the timeliness of on-board response; task reception and data downlink are both periodic or deterministic activities; all satellites in the system are isomorphic, carried The imaging load types are the same, and these simplifications and assumptions will make the practical application of these research results very difficult. 3) Most online scheduling algorithms are researched for non-agile satellites, and the research on agile satellite online scheduling algorithms is weak. The multi-agile autonomous satellite scheduling problem is a key problem that must be solved in 
the large-scale application of autonomous satellites in the future. At the same time, only a few scholars study the time-dependent timing constraints of the problem, which are constrained by time windows and time-dependent. The composition of the attitude and maneuver constraints is the key to restrict the efficiency of agile satellite applications. 4) Most of the current multi-satellite collaborative task scheduling research stays at the architectural design level, lacking detailed design of algorithm mechanisms for different architectures, and thus failing to propose the proposed architecture. Quantitative analysis verification does not form a feedback mechanism for architecture design. In addition, there are few studies on multi-star collaborative internal coordination control strategy, which leads to the failure to control the solution process of autonomous and collaborative scheduling problems. The degree of cooperation between modules is low. In order to meet the above-mentioned related research deficiencies, there is still much work to be done in the future of satellite autonomous and collaborative mission scheduling planning. 1) For satellite online scheduling, it is necessary to consider both the dynamic environment and computing power of the satellite. Delay in the delay of state information acquisition of other satellites, dynamic uncertain time difference between task release and task arrival, time availability of emergency tasks, and emergency response performance of on-board computing capability for dynamic tasks, etc., task planning/re-planning Decision time points, mechanism strategies, planning algorithms, etc., normalize the dynamic observation requirements. In addition, further in-depth research needs to consider the actions required to complete the task, not only to complete the task planning, but also to complete the corresponding action planning. 2) Future distributed satellites Most of the systems are heterogeneous, and the architecture of multi-star online collaboration is diverse. Different functional configuration methods form different collaborative architectures, and different collaborative architectures determine different collaborative algorithm mechanisms. Therefore, it is necessary to start from the specific collaborative algorithm design and evaluate the system performance and running cost of different collaborative architectures. In order to improve the cost-effectiveness ratio, feedback on the function configuration method is formed. 3) The essence of satellite independent planning technology is to transfer all the functions of planning and control originally undertaken by the ground system to the satellite. Therefore, the independent planning process is very Complex, not only need models and algorithms, but also rely on the coordination of various functional modules. Accurately divide the functional structure of the autonomous planning module, effectively decompose the complex autonomous planning problem solving process. Determine the coordination control strategy, control the independent planning problem Solve the process and achieve coordination between modules

In summary, satellite autonomous and collaborative task scheduling has broad research prospects, and its possible future development directions are:

1) Collaborative task scheduling for large-scale satellite clusters. Distributed Earth observation satellites currently applied. The scale of the system is small. With the expansion of satellite scale and the increase of load types, a single control structure will inevitably affect the cooperation efficiency of satellite clusters. The fully distributed constellation structure will be the development direction of large-scale satellite cluster cooperative mission planning. In the research, how to respond to a specific task with high quality in a large-scale satellite cluster, cooperate with multiple loads to complete the multi-method detection of the target, and keep the constellation stable, and become a collaborative task scheduling plan for studying large-scale satellite clusters key issues.

2) Close to the satellite scheduling plan that fully considers constraints. Most studies only carry out hypothesis experiments for a particular scenario, without considering many constraints in practical engineering applications, such as: regional target/moving target observation window changes ; uncertainty of energy and sequestration on the star; communication time window and communication loop pair Constraint of the letter, etc. With the development of satellite technology in the future, it is required to implement refined scheduling planning for satellites. The determination of specific time points needs to consider the scheduling of corresponding resources, and model and solve these scheduling sub-problems together. The full consideration of the constraints requires a new direction for satellite scheduling planning research. In addition, the on-board computing power is limited, how to improve the speed and efficiency of the algorithm under the consideration of complex constraints, is also the next research direction.

\section{CONFLICT OF INTEREST}

The authors declare no conflict of interest.

\section{AUTHOR CONTRIBUTIONS}

Jie Zhang conducted the research and wrote the paper; Gang Wang analyzed the data; Fangzheng Zhao collected study materials; Guiling Tian wrote the initial draft; all authors had approved the final version.

\section{ACKNOWLEDGMENT}

J. Z. thanks the Young Scientists Fund of the National Natural Science Foundation of China under Grant No. 61703412 and the China Postdoctoral Science Foundation under Grant No. 2016M602996.

\section{REFERENCES}

[1] G. Wu, et al., "A two-phase scheduling method with the consideration of task clustering for earth observing satellites," Computers \& Operations Research, vol. 40, no. 7, pp. 1884-1894, 2013.

[2] G. Wu, et al., "An adaptive simulated annealing-based satellite observation scheduling method combined with a dynamic task clustering strategy," Computer Science, 2014.

[3] P. Tangpattanakul, N. Jozefowiez, and P. Lopez, "A multiobjectivelocal search heuristic for scheduling Earth observation staken by an agile satellite," European Journal of Operational Research, vol. 245, no. 2, pp. 542-554, 2015.

[4] M. J. Sannella, "Constraint satisfaction and debugging for interactive user interfaces," doctoral thesis, University of Washington, 1994.

[5] V. Gabrel and D. Vanderpooten, "Enumeration and interactive eselection of efficient paths in a multiple criteria graph for scheduling 
an earth observing satellite," European Journal of Operational Research, vol. 139, no. 3, pp. 533-542, 2002.

[6] A. Sarkheyli, et al., "Scheduling earth observation activities in LEO satellites using graph coloring problem," in Proc. the 5th International Symposium on Telecommunications, 2010, pp. 928-931.

[7] S. Augenstein, "Optimal scheduling of Earth-imaging satellites with human collaboration via directed acyclic graphs," Journal of Experimental Psychology, vol. 38, no. 3, pp. 338-468, 2014.

[8] W. J. Wolfe and S. E. Sorensen, "Three scheduling algorithms applied to the earth observing systems domain," Management Science, vol. 46 no. 1 , p. 146,2000

[9] M. Vasquez and J. K. Hao, "A 'logic-constrained' knapsack formulation and a tabu algorithm for the daily photograph scheduling of an earth observation satellite," Computational Optimization \& Applications, vol. 20, no. 2, pp. 137-157, 2001.

[10] V. Gabrel and C. Murat, "Mathematical programming for earth observation satellite mission planning," Operations Research in Space and Air, pp. 103-122, 2003.

[11] S. Spangelo, et al., "Optimization-based scheduling for the single-satellite, multi-ground station communication problem," Computers \& Operations Research, vol. 57, pp. 1-16, 2015.

[12] P. Wang, et al., "A model, a heuristic and a decision support system to solve the scheduling problem of an earth observing satellite constellation," Computers \& Industrial Engineering, vol. 61, no. 2, pp. 322-33513, 2011.

[13] C. X. Ran, et al., "Study of electronic reconnaissance satellites mission scheduling model and algorithm," Communication Countermeasures, vol. 1105 , pp. 3-13, 2009
[14] Z. S. Gu and Y. W. Chen, "MIP model and algorithm for resolving scheduling of Earth observation satellites," Journal of Spacecraft TT\&C Technology, vol. 26, no. 1, pp. 19-24, 2007.

[15] X. W. Wang, Z. Chen, and C. Han, "Scheduling for single agile satellite, redundant targets problem using complex networks theory," Chaos Solitons \& Fractals, vol. 83, pp. 125-132, 2016.

[16] S. Chien, et al., "The Techsat-21 autonomous space science agent," in Proc. the International Joint Conference on Autonomous Agents and Multiagent Systems, 2002, pp. 570-577.

Copyright (C) 2020 by the authors. This is an open access article distributed under the Creative Commons Attribution License which permits unrestricted use, distribution, and reproduction in any medium, provided the original work is properly cited (CC BY 4.0).

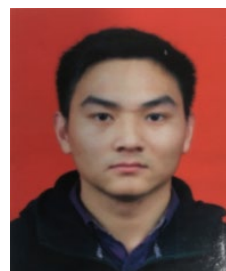

Jie Zhang was born on December 6, 1995. He is studying for a postgraduate degree. His research direction is combat multi-agent based on deep learning and tactical air defense and guidance command and control system. 